\title{
RESPONSE OF THE RAFT FOUNDATIONS UNDER DYNAMIC LOADS
}

\author{
E. A. El-Kasaby ${ }^{1}$, M. D. Khedr ${ }^{2}$, M. H. Mansour ${ }^{3}$, Mona I. Badawi ${ }^{4}$ \\ ${ }^{1}$ Prof. of soil mechanics and foundations. in Civil Engineering Department Benha Faculty of \\ Engineering, Benha University, Cairo, Egypt. \\ ${ }^{2}$ Lecturer. in Basic Engineering Sciences Department Benha Faculty of Engineering, Benha \\ University, Cairo, Egypt. \\ ${ }^{3}$ Lecturer in Civil Engineering Department Benha Faculty of Engineering, Benha University, \\ Cairo, Egypt. \\ ${ }^{4}$ Demonstrator in Civil Engineering Department Benha Faculty of Engineering, Benha \\ University, Cairo, Egypt.
}

\begin{abstract}
Modern industrial construction practice requires solutions to many problems concerning machine foundations. Modern manufacturing facilities have hammers, presses and turbines according to their production machinery. Vibrations due to machines may cause damage to structures nearby or even to machines themselves. These effects may extend to the surroundings and affect laborers and other sensitive machines within same place or neighboring residential areas. The Vibration amplitude and the forces transmitted to the supporting medium can become the governing factor of the foundation of these machines. So knowledge of the dynamic behavior of the foundation is important. The energy transmitted through soil and the response of the foundation system is essential for proper design of the system. This paper analyzes the response of a rigid raft foundation rested on the soil to eccentric harmonic, periodic and impulse forces (impact load).
\end{abstract}

\section{Arabic Abstract}

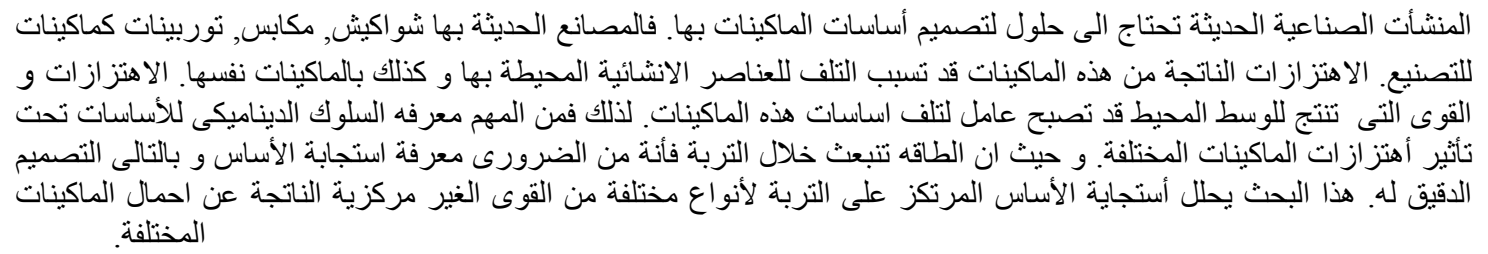

Keywords: response; raft foundations; dynamic stiffness; damping; shear modulus; Mathematica; Fourier expansion: harmonics.

\section{Introduction}

Foundations are supporting hammers, presses and turbines experience powerful dynamic and vibrating effects. The most important aspect which ensures the successful performance of machine is the proper dynamic behavior of the soilfoundation systems. The basic goal in the design of a machine foundation is to control its motion to amplitudes which will neither endanger the satisfactory operation of the machine nor will they disturb the workers in nearby areas. Thus, a key ingredient to a successful machine foundation design is the careful engineering analysis of the foundation response to the dynamic loads from the anticipated operation of the machine. Furthermore, when excessive motions of an existing foundation obstruct the operation of the supported machinery, analysis is necessary in order to understand the causes of the problem and hence to guide to appropriate remedial action.

\section{Lagrange's Equations of Motion}

The equation of motion of a vibrating system can often be derived in a simple manner in terms of generalized coordinates by the use of Lagrange's equations. Lagrange's equations can be stated, for an $n$ degree of freedom system, as

$$
\frac{d}{d t}\left(\frac{\partial T}{\partial q_{j}}\right)-\frac{\partial T}{\partial q_{j}}+\frac{\partial V}{\partial q_{j}}=Q_{j}^{(n)} \quad, j=1,2, \ldots . ., \mathrm{n}
$$

Where $\tilde{q}_{j}=\frac{\partial q_{j}}{\partial t}$ is the generalized velocity and 
$Q_{j}^{(n)}$ is the non-conservative generalized force corresponding to the generalized coordinate $q_{j}$. The forces represented by $Q_{j}^{(n)}$ may be dissipative (damping) forces or other external forces that are not derivable from a potential function, if $F_{x k}$, $F_{y k}$ and $F_{z k}$ represent the external forces acting on the $\mathrm{k}^{\text {th }}$ mass of the system in the $\mathrm{x}, \mathrm{y}$ and $\mathrm{z}$ directions, respectively, then the generalized force $Q_{j}^{(n)}$ can be computed as follows

$Q_{j}^{(n)}=\Sigma_{k}\left(\mathrm{~F}_{\mathrm{xk}} \frac{\partial x_{\mathrm{k}}}{\partial q_{j}}+\mathrm{F}_{\mathrm{yk}} \frac{\partial y_{k}}{\partial q_{j}}+\mathrm{F}_{\mathrm{zk}} \frac{\partial z_{\mathrm{k}}}{\partial q_{j}}\right)$

Where $x_{k}, y_{k}$ and $z_{k}$ are the displacements of the $\mathrm{k}^{\text {th }}$ mass in $\mathrm{x}, \mathrm{y}$ and $\mathrm{z}$ directions, respectively. Note that for a torsional system, the force $F_{x k}$, for example, is to be replaced by the moment acting about the $\mathrm{x}$ axis $\left(M_{x k}\right)$, and the displacement $x_{k}$ by the angular displacement about $\mathrm{x}$ axis $\left(\theta_{x k}\right)$ in the previous equation. For a conservative system $Q_{j}^{(n)}$ $=0$, so the equation takes the form

$\frac{d}{d t}\left(\frac{\partial T}{\partial q_{j}}\right)-\frac{\partial T}{\partial q_{j}}+\frac{\partial V}{\partial q_{j}}=0, \quad j=1,2, \ldots, \mathrm{n}$

The dynamic stiffness of soil, evaluated by more methods, depends on the exciting force, if the force is periodic. The free body diagram of the system. The dynamic stiffness has been calculated as follows:

$\sum f=m a, \quad \mathrm{p}(\mathrm{t})-\mathrm{R}(\mathrm{t})=\mathrm{m} \tilde{u}$

Force-displacement relation

$\mathrm{R}(\mathrm{t})=\mathrm{ku}$

Substituting in equation (3) [1]

$\mathrm{m} \bar{u}+\mathrm{ku}=\mathrm{p}(\mathrm{t})$

This equation can be used if the damping is neglected. But if the damping is used

$f_{c}=\mathrm{cu}$ [1] thus equation (6) can be written as

$\mathrm{m} \ddot{u}+\mathrm{ku}+\mathrm{c} \tilde{u}=\mathrm{p}(\mathrm{t})$

The equation of motion of the block can be written in the matrix form as:

$[m] \vec{q} \vec{q}+[c] \vec{q}+[k] \vec{q}=\vec{f}(\mathrm{t})$

Where $\vec{q}$ is the generalized coordinate vector.

$\vec{q}=\left[\begin{array}{l}z \\ \theta \\ \varnothing\end{array}\right],[m]$ is the mass matrix, $[c]$ is the damping matrix and $[k]$ is the stiffness matrix, $\overrightarrow{f_{t}}$ is the force vector.

$q_{1}=\mathrm{z} ; \quad q_{2}=; \quad q_{a}=\emptyset$

Potential and Kinetic Energy Expressions in Matrix Form

Let $x_{i}$ denotes the displacement of mass $m_{i}$ and $F_{i}$ the force applied in the direction of $x_{i}$ at mass $m_{\mathrm{i}}$ in an $n$ degree of freedom system. The elastic potential energy (also known as strain energy or energy of deformation) of the $\mathrm{i}^{\text {th }}$ springs is given by:
$V_{\mathrm{i}}=\frac{1}{2} F_{\mathrm{i}} x_{\mathrm{i}}$

The total potential energy can be expressed as

$\mathrm{V}=\sum_{\mathrm{i}=1}^{\mathrm{n}} V_{\mathrm{i}}=\frac{1}{2} \sum_{\mathrm{i}=1}^{\mathrm{n}} F_{\mathrm{i}} x_{\mathrm{i}}$

Since

$F_{i}=\sum_{j=1} k_{i j} x_{j}$

$\mathrm{V}=\frac{1}{2} \sum_{i=1}^{n}\left(\sum_{j=1}^{n} k_{i j} x_{j}\right) x_{i}$

This equation can be written in matrix form as

$\mathrm{V}=\frac{1}{2} \overrightarrow{x^{\mathrm{t}}}[K] \vec{x}$

Where the displacement vector and the stiffness matrix are given by

$\vec{x}=\left\{\begin{array}{c}x_{1}(t) \\ x_{2}(t) \\ \vdots \\ \vdots \\ x_{n}(t)\end{array}\right\},[K]=\left[\begin{array}{cccc}k_{11} & k_{12} & \cdots & k_{1 n} \\ k_{21} & k_{22} & \cdots & k_{2 n} \\ \vdots & & & \\ k_{n 1} & k_{n 2} & \cdots & k_{n n}\end{array}\right]$

The kinetic Energy Associated with Mass $m_{i}$ is, by Definition Equal to

$T_{\mathrm{i}}=\frac{1}{2} m_{\mathrm{i}} \dot{x}_{\mathrm{i}}^{2}$

The total kinetic energy of the system can be expressed as

$\mathrm{T}=\sum_{\mathrm{i}=1}^{\mathrm{n}} T_{\mathrm{i}}=\frac{1}{2} \sum_{\mathrm{i}=1}^{\mathrm{n}} m_{\mathrm{i}} \dot{x}_{\mathrm{i}}^{2}$

This can be written in matrix form as

$\mathrm{T}=\frac{1}{2} \frac{\vec{x}}{\vec{x}}[\mathrm{~m}] \overrightarrow{\vec{x}}$

Where the velocity vector $\vec{x}$ and the mass matrix are given by

$\overrightarrow{\vec{x}}=\left\{\begin{array}{c}\dot{x}_{1} \\ \dot{x}_{2} \\ \vdots \\ \dot{x}_{n}\end{array}\right\},[m]=\left[\begin{array}{cccc}m_{11} & m_{12} & \cdots & m_{1 n} \\ m_{21} & m_{22} & & m_{2 n} \\ \vdots & & & \vdots \\ m_{n 1} & m_{n 2} & \cdots & m_{n n}\end{array}\right]$

\section{Mathematical model}

The analysis of machines foundation is usually performed by idealizing it as a simple system as shown in Fig. 1. which shows a schematic sketch of a rigid concrete block (Raft) resting on the ground surface and supporting machines. Let us assume that the operation of the machines produce a vertical eccentric force which doesn't pass through the combined centre of gravity of the machines foundation system but make two eccentricities $e_{x}, e_{y}$ from $\mathrm{y}$ and $\mathrm{x}$ axes respectively. Under this condition, the foundation will vibrate in the vertical direction about its position and rotate about $\mathrm{x}$ axes by angle $\theta$ and about y axes by angle $\phi$. The vibration of the foundation results in transmission of waves through the soil. These waves carry energy with them. This loss of energy is termed "Geometrical damping". The soil below the footing experiences cyclic deformations and absorbs some energy which is termed "material damping". The material damping is generally small compared to the geometrical damping and may be neglected in most cases. However, material damping may also 
become important in some cases of machine foundation vibrations. The problem of a rigid block foundation resting on the ground surface, Fig. 2. may therefore be represented in a responsible manner by a spring-mass-dashpot system shown in Fig. 2a. the spring in this figure is the equivalent soil spring which represents the elastic resistance of the soil below the base of the foundation. The dashpot represents the energy loss or the damping effect. The mass shown in Fig. 2b. is the mass of the foundation block and the machines. If damping is neglected, a spring-mass system shown in Fig 2c may be used to represent the problem defined in Fig. 1. Three degree of freedom model shown in Fig. 1. may in fact be used to represent the problem in machine foundation vibration in any mode of vibration if appropriate values of equivalent soil spring and damping constants are used.

\subsection{Force Time Relation from a Steam Turbine Machine}

The steam turbine machine is a rotary machine. The type of dynamic loading in soil or the foundations of a structure depend on the natural frequency of the source producing it[3]. The force from a Steam Turbine Machine will be in formula as a function of time as shown in Fig. 3.

Where The force from the steam turbine machine is a harmonic force as:

$$
F(t)=F_{0} \cos \omega t
$$

For such a harmonic forcing function, the nonhomogeneous solution can be easily obtained using the theory of ordinary differential equations. However, the same can also be solved using vectorial representation of all the forces acting on the system. The particular solution in this case can be assumed as

$$
x_{p}(t)=X \cos (\omega t-\emptyset)
$$

Where $\mathrm{X}$ is the amplitude and $\varphi$ is the phase angle. for $X$ and $\emptyset$ by substituting the above solution in the differential equation of motion given by equation (8) that is inertia force + damping force + spring force + external force $=0$

These forces can be vectorially represented as shown in Fig. 4. from this vector

Diagram $X$ and $\emptyset$ can be readily obtained as

$$
X=\frac{F_{0} / k}{\sqrt{\left(1-\left(\frac{\omega}{\omega}\right)^{2}\right)^{2}+\left(2 \zeta \frac{\omega}{\omega_{n}}\right)^{2}}}
$$

And

$$
\begin{aligned}
& \tan \emptyset=\frac{2 \zeta \frac{\omega}{\omega_{n}}}{1-\left(\frac{\omega}{\omega_{n}}\right)^{2}}=\frac{2 \zeta r}{1-r^{2}} \\
& \mathrm{M}=\frac{X}{F_{0} / k}=\frac{X}{B_{s t}}=\frac{1}{\left[\left(1-\left(\frac{\omega}{w_{n}}\right)^{2}\right)^{2}+\left(2 \zeta \frac{\omega}{\omega_{n}}\right)^{2}\right]^{1 / 2}}= \\
& \frac{1}{\left[\left(1-r^{2}\right)^{2}+(2 \zeta r)^{2}\right]^{1 / 2}}
\end{aligned}
$$

Where $\delta_{s t}=F_{0} / k$ the static deflection under force $F_{0}$.

$\zeta=\frac{c}{2 m \omega_{n}}=\frac{c}{2 \sqrt{k m}}$

Where $\mathrm{c}$ is the damping coefficient, $\mathrm{m}$ is the mass of foundation and machine,

$\omega_{n}$ is the Natural frequency and $\mathrm{k}$ is the stiffness coefficient.

\subsection{Force Time Relation from Mechanical Presses}

The mechanical press is an impact machine which produces periodic force. The type of dynamic loading in soil or the foundations of a structure depend on the natural frequency of the source producing it[3]. The force from mechanical press will be in formula as a function of time as in Fig.5. Any periodic function of time can be represented by Fourier series as an infinite sum of Sine and Cosine functions

If $f(t)$ is a periodic function with period $\tau=\frac{2 \pi}{\omega}$ its

Fourier series representation is given by

$F(t)=\frac{a_{0}}{2}+\sum_{j=1}^{m} a_{j} \cos j \omega t+\sum_{j=1}^{m} a_{j} \sin j \omega t$

Where

$a_{j}=\frac{2}{\tau} \int_{0}^{\tau} F(t) \cos j \omega t d t \quad j j=0,1,2, \ldots$

$b_{j}=\frac{2}{\tau} \int_{0}^{\tau} F(t) \sin j \omega t d t \quad, j=1,2,3, \ldots$

$x(t)=\frac{a_{0}}{2 k}+\sum_{j=1}^{\infty} \frac{\left(\frac{a_{1}}{k}\right)}{\sqrt{\left(1-j^{2} r^{2}\right)^{2}+(2 \zeta j y)^{2}}} \cos \left(j \omega t-\emptyset_{j}\right)$

$+\sum_{j=1}^{=\infty} \frac{\left(\frac{b_{1}}{k}\right)}{\sqrt{\left(1-j^{2} r^{2}\right)^{2}+(2 \zeta j p)^{2}}} \sin \left(j \omega t-\emptyset_{j}\right)[4]$ 


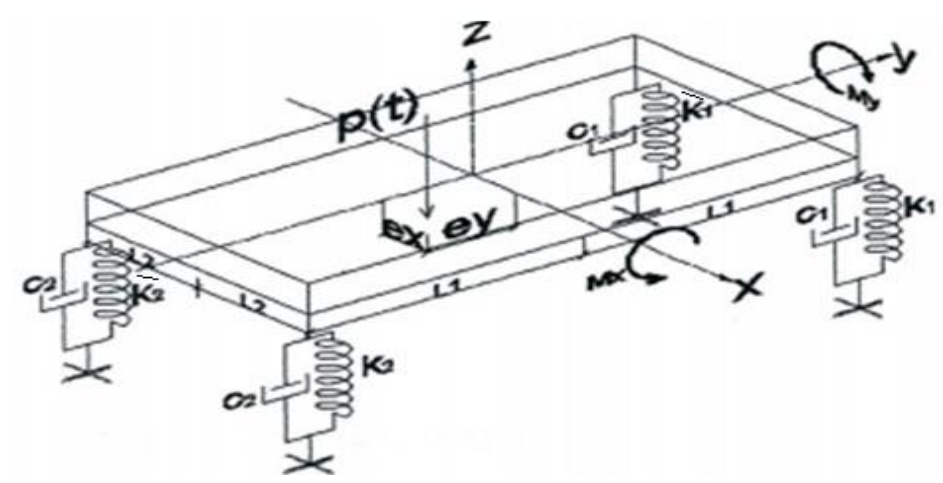

Fig. 1. The model
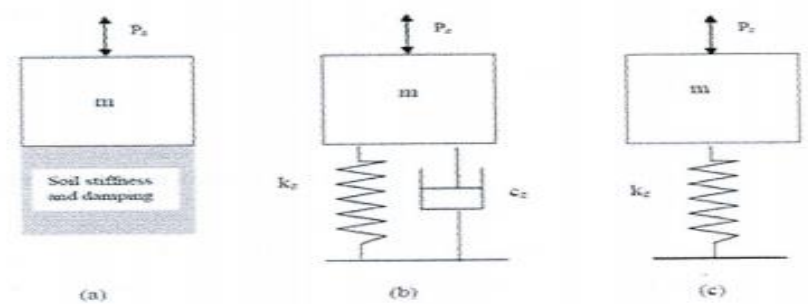

Fig. 2. Spring-mass-dashpot system

\subsection{Force Time Relation from Forging Hammer}

The forging hammer is an impact machine which produces an impact load. The type of dynamic loading in soil or the foundations of a structure depend on the nature frequency of the source producing it [3]. The force from forging hammer will be chosen in formula as a function of time as in Fig. 6.

It is a half-sine impulsive load. This force can be expressed as a function of time as

$$
f(t)=\left\{\begin{array}{cc}
\left(P_{g} \sin \left(\frac{\pi}{t_{p}} t\right)=P_{g} \sin (\omega t),\right. & 0<t<t_{P} \\
0_{v} & t>t_{P}
\end{array}\right.
$$

Where $\omega$ is the frequency produced from machine

$$
\begin{aligned}
& x(t)=\frac{\frac{p_{r} \omega}{m}}{\left(\omega^{2}-\omega_{n}^{2}\right)^{2}+4 \zeta^{2} \omega^{2} \omega_{n}^{2}}\left[-2 \zeta \omega_{n} \cos (\omega t)+\frac{\omega^{2}-\omega_{n}^{2}}{\omega} \sin \omega t\right. \\
& \left.+2 \zeta \omega_{n} \mathrm{e}^{-\zeta \omega_{n} t} \cos \left(\omega_{n}^{g} t\right)+\left(\frac{\omega^{2}-\omega_{n}^{2}+4 \zeta \omega_{n}^{2}}{\omega_{n}^{t}}\right) e^{-\zeta \omega_{n} t} \sin \left(\omega_{n}^{g} t\right)\right]
\end{aligned}
$$

Where

$$
\omega_{n}=\sqrt{\frac{k}{m}}, \omega_{n}^{0}=\omega_{n} \sqrt{1-\zeta^{2}}, \zeta=\frac{c}{2 \sqrt{m k}}[1]
$$

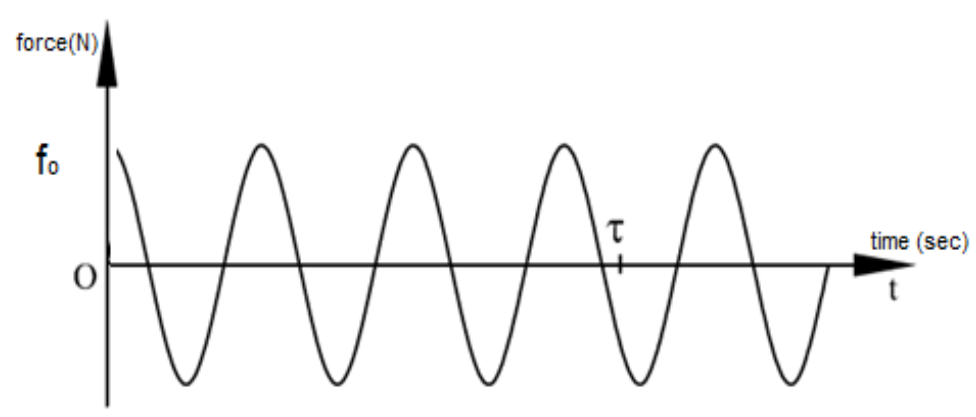

Fig. 3. Force time relation from steam turbine

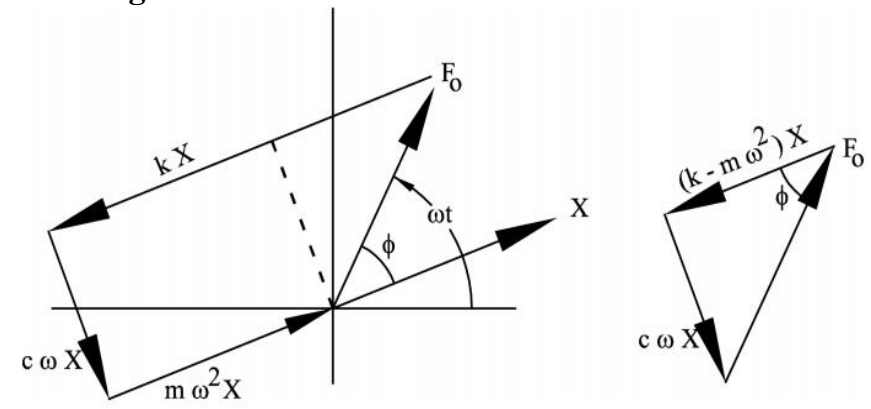

Fig. 4.Vector representation of forced vibration with viscous damping 


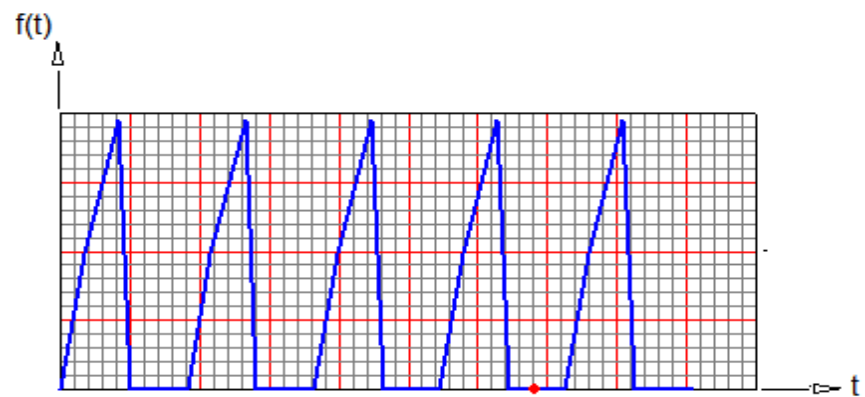

Fig. 5. Force-time relation from press

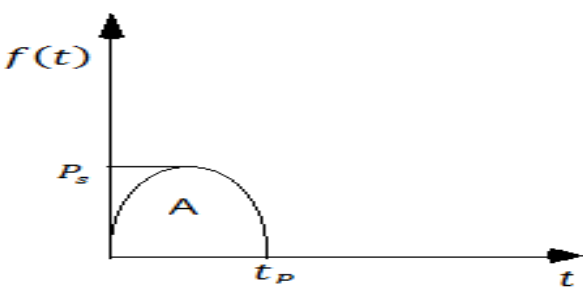

Fig. 6.Force-time relation from forging hammer

\subsection{Modeling of the Problem}

3.4.1 Potential energy of the system can be expressed as

$\mathrm{V}=\frac{1}{2} \vec{q}^{T}[\mathrm{k}] \vec{q}$

$$
\begin{aligned}
& \mathrm{V} \quad=\quad \frac{1}{2} k_{1}\left[z+l_{1} \theta-l_{2} \varnothing\right]^{2} \\
& \frac{1}{2} \bar{k}_{1}\left[z+l_{1} \theta+l_{2} \varnothing\right]^{2}+\frac{1}{2} k_{2}\left[z-l_{1} \theta-l_{2} \varnothing\right]^{2}+ \\
& \frac{1}{2} \bar{k}_{2}\left[z-l_{1} \theta+l_{2} \varnothing\right]^{2} \\
& \mathrm{~V} \quad(32) \quad \frac{1}{2} z^{2}\left[k_{1}+\bar{k}_{1}+k_{2}+\bar{k}_{2}\right]_{+} \\
& \frac{1}{2} \theta^{2}\left[k_{1} l_{1}^{2}+\bar{k}_{1} l_{1}^{2}+k_{2} l_{1}^{2}+\bar{k}_{2} l_{1}^{2}\right]_{+} \\
& \frac{1}{2} \emptyset^{2}\left[k_{1} l_{2}^{2}+\bar{k}_{1} l_{2}^{2}+k_{2} l_{2}^{2}+\bar{k}_{2} l_{2}^{2}\right]_{+} \\
& \frac{1}{2} z \theta\left[2 k_{1} l_{1}+2 \bar{k}_{1} l_{1}-2 k_{2} l_{1}-2 \bar{k}_{2} l_{1}\right]+ \\
& \frac{1}{2} z \emptyset\left[-2 k_{1} l_{2}+2 \bar{k}_{1} l_{2}-2 k_{2} l_{2}+2 \bar{k}_{2} l_{2}\right]+ \\
& \frac{1}{2} \theta \varnothing\left[-2 k_{1} l_{1} l_{2}+2 \bar{k}_{1} l_{1} l_{2}+2 k_{2} l_{1} l_{2}-2 \bar{k}_{2} l_{1} l_{2}\right]
\end{aligned}
$$

The stiffness matrix can be written in the form as equation (41)

\subsubsection{Rayleigh's dissipation function of the} system can be expressed as

$$
\begin{aligned}
& \mathrm{R}=\frac{1}{2} \overrightarrow{q^{T}}[\mathrm{c}] \vec{q} \\
& \mathrm{R}=\frac{1}{2} c_{1}\left[\tilde{z}+l_{1} \theta-l_{2} \dot{\phi}\right]^{2}+ \\
& \frac{1}{2} \bar{l}_{1}\left[\tilde{z}+l_{1} \theta+l_{2} \dot{\phi}\right]^{2}+\frac{1}{2} c_{2}\left[\tilde{z}-l_{1} \theta-l_{2} \dot{\phi}\right]^{2}+ \\
& \frac{1}{2} \bar{c}_{2}\left[z-l_{1} \dot{z}+l_{2} \dot{\phi}\right]^{2}
\end{aligned}
$$

$$
\begin{aligned}
& \mathrm{R} \quad=\quad \frac{1}{2} z^{2}\left[c_{1}+\bar{c}_{1}+c_{2}+\bar{c}_{2}\right]+ \\
& \frac{1}{2} \theta^{2}\left[c_{1} l_{1}^{2}+\bar{c}_{1} l_{1}^{2}+c_{2} l_{1}^{2}+\bar{c}_{2} l_{1}^{2}\right]_{+} \\
& \frac{1}{2} \Phi^{2}\left[c_{1} l_{2}^{2}+\bar{c}_{1} l_{2}^{2}+c_{2} l_{2}^{2}+\bar{c}_{2} l_{2}^{2}\right]_{+} \\
& \frac{1}{2} \theta\left[2 c_{1} l_{1}+2 \bar{c}_{1} l_{1}-2 c_{2} l_{1}-2 \bar{c}_{2} l_{1}\right]_{+} \\
& \frac{1}{2} \approx \Phi\left[-2 c_{1} l_{2}+2 \bar{c}_{1} l_{2}-2 c_{2} l_{2}+2 \bar{c}_{2} l_{2}\right]+ \\
& \frac{1}{2} \theta \phi\left[-2 c_{1} l_{1} l_{2}+2 \bar{c}_{1} l_{1} l_{2}+2 c_{2} l_{1} l_{2}-2 \bar{c}_{2} l_{1} l_{2}\right]
\end{aligned}
$$

The damping matrix can be written in the form as in equation (42)

3.4.3 Kinetic energy of the system can be expressed as

$\mathrm{T}=\frac{1}{2} \frac{\vec{q}}{q}[\mathrm{k}] \stackrel{\vec{q}}{q}$

$\mathrm{T}=\frac{1}{2} m \dot{z}^{2}+\frac{1}{2} J_{x} \theta^{2}+\frac{1}{2} J_{y} \Phi^{2}$

the mass can be written in matrix form as:

$$
[m]=\left[\begin{array}{ccc}
m & 0 & 0 \\
0 & J_{x} & 0 \\
0 & 0 & J_{y}
\end{array}\right]
$$

The force vector can be obtained from the principle of virtual work

$$
\begin{aligned}
& \delta w=-P\left(\delta z-\delta e_{y} \theta-\delta e_{x} \emptyset\right)= \\
& -P \delta z+P \delta e_{y} \theta+P \delta e_{x} \emptyset
\end{aligned}
$$

Where; $M_{x}=P .\left(e_{y}\right), M_{y}=P \cdot\left(e_{x}\right)$

That can be written in matrix form as

$$
[f]=\left[\begin{array}{l}
-p_{t} \\
M_{x} \\
M_{y}
\end{array}\right]
$$

Substituting in equation (8) from equations (38), (41), (42) and (40) 


$$
\begin{array}{r}
{[k]=\left[\begin{array}{ccc}
{\left[k_{1}+\bar{k}_{1}+k_{2}+\bar{k}_{2}\right]} & {\left[k_{1} l_{1}+\bar{k}_{1} l_{1}-k_{2} l_{1}-\bar{k}_{2} l_{1}\right]} & {\left[-k_{1} l_{2}+\bar{k}_{1} l_{2}-k_{2} l_{2}+\bar{k}_{2} l_{2}\right]} \\
{\left[k_{1} l_{1}+\bar{k}_{1} l_{1}-k_{2} l_{1}-\bar{k}_{2} l_{1}\right]} & {\left[k_{1} l_{1}^{2}+\bar{k}_{1} l_{1}^{2}+k_{2} l_{1}^{2}+\bar{k}_{2} l_{1}^{2}\right.} & {\left[-k_{1} l_{1} l_{2}+\bar{k}_{1} l_{1} l_{2}+k_{2} l_{1} l_{2}-\bar{k}_{2} l_{1} l_{2}\right]} \\
{\left[-k_{1} l_{2}+\bar{k}_{1} l_{2}-k_{2} l_{2}+\bar{k}_{2} l_{2}\right]} & {\left[-k_{1} l_{1} l_{2}+\bar{k}_{1} l_{1} l_{2}+k_{2} l_{1} l_{2}-\bar{k}_{2} l_{1} l_{2}\right]} & {\left[k_{1} l_{2}^{2}+\bar{k}_{1} l_{2}^{2}+k_{2} l_{2}^{2}+\bar{k}_{2} l_{2}^{2}\right]}
\end{array}\right]} \\
{[c]=\left[\begin{array}{ccc}
{\left[c_{1}+\bar{c}_{1}+c_{2}+c_{2}\right]} & {\left[c_{1} l_{1}+\bar{c}_{1} l_{1}-c_{2} l_{1}-\bar{c}_{2} l_{1}\right]} & {\left[-c_{1} l_{2}+\bar{c}_{1} l_{2}-c_{2} l_{2}+\bar{c}_{2} l_{2}\right]} \\
{\left[c_{1} l_{1}+\bar{c}_{1} l_{1}-c_{2} l_{1}-c_{2} l_{1}\right]} & {\left[c_{1} l_{1}^{2}+\bar{c}_{1} l_{1}^{2}+c_{2} l_{1}^{2}+\bar{c}_{2} l_{1}^{2}\right]} & {\left[-c_{1} l_{1} l_{2}+\bar{c}_{1} l_{1} l_{2}+c_{2} l_{1} l_{2}-\bar{c}_{2} l_{1} l_{2}\right]} \\
{\left[-c_{1} l_{2}+\bar{c}_{1} l_{2}-c_{2} l_{2}+\bar{c}_{2} l_{2}\right]} & {\left[-c_{1} l_{1} l_{2}+\bar{c}_{1} l_{1} l_{2}+c_{2} l_{1} l_{2}-c_{2} l_{1} l_{2}\right]} & {\left[c_{1} l_{2}^{2}+c_{1} l_{2}^{2}+c_{2} l_{2}^{2}+\bar{c}_{2} l_{2}^{2}\right]}
\end{array}\right]}
\end{array}
$$

$$
\begin{aligned}
& {\left[\begin{array}{ccc}
m & 0 & 0 \\
0 & J_{x} & 0 \\
0 & 0 & J_{y}
\end{array}\right]\left[\begin{array}{l}
\tilde{z} \\
\ddot{\theta} \\
\ddot{\emptyset}
\end{array}\right]+\left[\begin{array}{ccc}
{\left[c_{1}+\bar{c}_{1}+c_{2}+c_{2}\right]} & {\left[c_{1} l_{1}+\bar{c}_{1} l_{1}-c_{2} l_{1}-\bar{c}_{2} l_{1}\right]} & {\left[-c_{1} l_{2}+\bar{c}_{1} l_{2}-c_{2} l_{2}+\bar{c}_{2} l_{2}\right]} \\
{\left[c_{1} l_{1}+\bar{c}_{1} l_{1}-c_{2} l_{1}-c_{2} l_{1}\right]} & {\left[c_{1} l_{1}^{2}+\bar{c}_{1} l_{1}^{2}+c_{2} l_{1}^{2}+\bar{c}_{2} l_{1}^{2}\right]} & {\left[-c_{1} l_{1} l_{2}+\bar{c}_{1} l_{1} l_{2}+c_{2} l_{1} l_{2}-\bar{c}_{2} l_{1} l_{2}\right]} \\
{\left[-c_{1} l_{2}+\bar{c}_{1} l_{2}-c_{2} l_{2}+\bar{c}_{2} l_{2}\right]} & {\left[-c_{1} l_{1} l_{2}+\bar{c}_{1} l_{1} l_{2}+c_{2} l_{1} l_{2}-c_{2} l_{1} l_{2}\right]} & {\left[c_{1} l_{2}^{2}+c_{1} l_{2}^{2}+c_{2} l_{2}^{2}+\bar{c}_{2} l_{2}^{2}\right]}
\end{array}\right]\left[\begin{array}{l}
\tilde{z} \\
\hat{\theta} \\
\dot{\emptyset}
\end{array}\right] } \\
&+\left[\begin{array}{ccc}
{\left[k_{1}+\bar{k}_{1}+k_{2}+\bar{k}_{2}\right]} & {\left[k_{1} l_{1}+\bar{k}_{1} l_{1}-k_{2} l_{1}-\bar{k}_{2} l_{1}\right]} & {\left[-k_{1} l_{2}+\bar{k}_{1} l_{2}-k_{2} l_{2}+\bar{k}_{2} l_{2}\right]} \\
{\left[k_{1} l_{1}+\bar{k}_{1} l_{1}-k_{2} l_{1}-\bar{k}_{2} l_{1}\right]} & {\left[k_{1} l_{1}^{2}+\bar{k}_{1} l_{1}^{2}+k_{2} l_{1}^{2}+\bar{k}_{2} l_{1}^{2}\right]} & {\left[-k_{1} l_{1} l_{2}+\bar{k}_{1} l_{1} l_{2}+k_{2} l_{1} l_{2}-\bar{k}_{2} l_{1} l_{2}\right]} \\
{\left[-k_{1} l_{2}+\bar{k}_{1} l_{2}-k_{2} l_{2}+\bar{k}_{2} l_{2}\right]} & {\left[-k_{1} l_{1} l_{2}+\bar{k}_{1} l_{1} l_{2}+k_{2} l_{1} l_{2}-\bar{k}_{2} l_{1} l_{2}\right]} & {\left[k_{1} l_{2}^{2}+\bar{k}_{1} l_{2}^{2}+k_{2} l_{2}^{2}+\bar{k}_{2} l_{2}^{2}\right]}
\end{array}\right]=\left[\begin{array}{l}
-p_{t} \\
M_{x} \\
M_{y} \\
M_{y}
\end{array}\right]
\end{aligned}
$$

From equation (43) we can derive the following equations $m \ddot{z}+\left[c_{1}+\bar{c}_{1}+c_{2}+c_{2}\right] z^{*}+\left[c_{1} l_{1}+\bar{c}_{1} l_{1}-c_{2} l_{1}-\bar{c}_{2} l_{1}\right] \theta+$ $\left[-c_{1} l_{2}+\bar{c}_{1} l_{2}-c_{2} l_{2}+\bar{c}_{2} l_{2}\right] \emptyset+\left[k_{1}+\bar{k}_{1}+k_{2}+\bar{k}_{2}\right] z+$ $\left[k_{1} l_{1}+\bar{k}_{1} l_{1}-k_{2} l_{1}-\bar{k}_{2} l_{1}\right] \theta+\left[-k_{1} l_{2}+\bar{k}_{1} l_{2}-k_{2} l_{2}+\right.$ $\left.\bar{k}_{2} l_{2}\right] \varnothing=-p_{t}$

$l_{x} \bar{\theta}+\left[c_{1} l_{1}+\bar{c}_{1} l_{1}-c_{2} l_{1}-c_{2} l_{1}\right] \bar{z}+\left[c_{1} l_{1}^{2}+\bar{c}_{1} l_{1}^{2}+c_{2} l_{1}^{2}+\right.$ $\left.\bar{c}_{2} l_{1}^{2}\right] \theta+\left[-c_{1} l_{1} l_{2}+\bar{c}_{1} l_{1} l_{2}+c_{2} l_{1} l_{2}-\bar{c}_{2} l_{1} l_{2}\right] \emptyset+$ $\left[k_{1} l_{1}+\bar{k}_{1} l_{1}-k_{2} l_{1}-\bar{k}_{2} l_{1}\right] z+\left[k_{1} l_{1}^{2}+\bar{k}_{1} l_{1}^{2}+k_{2} l_{1}^{2}+\right.$ $\left.\bar{k}_{2} l_{1}^{2}\right] \theta+\left[-k_{1} l_{1} l_{2}+\bar{k}_{1} l_{1} l_{2}+k_{2} l_{1} l_{2}-\bar{k}_{2} l_{1} l_{2}\right] \emptyset=M_{x}$

$l_{y} \ddot{\Phi}\left[-c_{1} l_{2}+\bar{c}_{1} l_{2}-c_{2} l_{2}+\right.$ $\left.\bar{c}_{2} l_{2}\right] \tilde{z}+\left[-c_{1} l_{1} l_{2}+\bar{c}_{1} l_{1} i_{2}+c_{2} l_{1} l_{2}-c_{2} l_{1} l_{2}\right] \theta+$ $\left[c_{1} l_{2}^{2}+c_{1} l_{2}^{2}+c_{2} l_{2}^{2}+\bar{c}_{2} l_{2}^{2}\right] \emptyset+\left[-k_{1} l_{2}+\bar{k}_{1} l_{2}-k_{2} l_{2}+\right.$ $\left.\bar{k}_{2} l_{2}\right] z+\left[-k_{1} l_{1} l_{2}+\bar{k}_{1} l_{1} l_{2}+k_{2} l_{1} l_{2}-\bar{k}_{2} l_{1} l_{2}\right] \theta+$ $\left[k_{1} l_{2}^{2}+\bar{k}_{1} l_{2}^{2}+k_{2} l_{2}^{2}+\bar{k}_{2} l_{2}^{2}\right] \emptyset=M_{y}$

\section{Numerical examples}

\subsection{Case1}

The foundation is subjected to an eccentric harmonic force and rests on a homogenous soft clay soil (the stiffness and damping at all corners are equal)

This soft clay has properties $\Phi=0, \mathrm{C}=40 \mathrm{KN} / \mathrm{m}^{2}, \rho$ $=18 \mathrm{KN} / \mathrm{m}^{2}, \mathrm{E}=15 \mathrm{Mpa}[5], \mathrm{G}=\frac{E}{2(1+\mathrm{m})}=\frac{15 \times 10^{6}}{2(1+0.5)}$ $=5 M p a, \omega=314.2 \mathrm{rad} / \mathrm{sec}$

Dynamic soil properties are:

$$
\overline{k_{\mathrm{l}}}=n_{\mathrm{i}} \times k_{\mathrm{i}} \quad, \quad \overline{c_{\mathrm{i}}}=\lambda_{\mathrm{i}} \cdot c_{\mathrm{i}}[6]
$$$$
k_{z}=S_{z} \frac{2 L G}{1-\mathbb{I}}, S_{z}=0.73+1.54\left(l_{a}\right)^{0.75}=0.73+1.54(1)^{0.75}
$$

$=2.27$

$k_{z}=2.27 \times \frac{2 \times 7.5 \times 5000000}{1-0.5}=340500000 \mathrm{~N} / \mathrm{m}$

$c_{z}=\rho \cdot V_{L A^{*}} A=18000 \times 26.37 \times 15 \times 15=106798500$ $\mathrm{kg} / \mathrm{m}$

$k_{d}=\overline{k_{z}}-\omega \overline{c_{z}} D_{z}=340500000-314.2 \times 106798500 \times 0.005$ $=172719557 \mathrm{~N} / \mathrm{m}$ $c_{d}=\overline{c_{z}}+\frac{2 \overline{k_{z}} D_{z}}{\omega}$

$=106798500+\frac{2 \times 181.827 \times 10^{6} \times 0.005}{714.2}=$ $106804287 \mathrm{~kg} / \mathrm{sec}$

For machine:

Weight of Machine $=250$ ton

For rigid Raft foundation :

The dimension of the footing is $15 \times 15 \mathrm{~m}$ assume that the net allowable stress of soil $q_{n a}=0.5 \mathrm{~kg} / \mathrm{cm}^{2}$. Assume the thickness of foundation $=1.5 \mathrm{~m}[7]$

1 - Mass of foundation $=(337.5 \times 2500)+250000=1093750$ $\mathrm{kg}$

2- $I_{x}$ Mass moment of inertia about $\mathrm{x}$ axis

$$
f_{x}=\frac{1}{12} m\left(x^{2}+z^{2}\right)=248554688 \mathrm{~kg} \cdot \mathrm{m}^{2}
$$

3- $f_{y}$ Mass moment of inertia about y axis

$$
l_{y}=\frac{1}{12} m\left(y^{2}+z^{2}\right)=248554688 \mathrm{~kg} \cdot \mathrm{m}^{2}
$$

Force properties

The force is a harmonic of the form $\mathrm{F}=F_{0} \cos \omega t$. The force is eccentric from the center of the foundation $e_{x}=1 m_{s} e_{y}=0.5 m, F_{0}=963000 \mathrm{~N}, \omega=3000 \mathrm{rpm}$ Substituting in the general form equation(47)

$$
\begin{aligned}
& {\left[\begin{array}{ccc}
1093750 & 0 & 0 \\
0 & 248554688 & 0 \\
0 & 0 & 248554688
\end{array}\right]\left[\begin{array}{l}
\tilde{z} \\
\tilde{\theta} \\
\ddot{\theta}
\end{array}\right]} \\
& +\left[\begin{array}{ccc}
427217148 & 0 & 0 \\
0 & 2.4030965 \times 10^{20} & 0 \\
0 & 0 & 2.4030965 \times 10^{20}
\end{array}\right]\left[\begin{array}{l}
\tilde{z} \\
\tilde{\theta} \\
\tilde{\theta}
\end{array}\right] \\
& +\left[\begin{array}{ccc}
690878228 & 0 & 0 \\
0 & 3.886191 \times 10^{10} & 0 \\
0 & 0 & 3.886191 \times 10^{10}
\end{array}\right] \|\left[\begin{array}{l}
\tilde{z} \\
\theta \\
0
\end{array}\right] \\
& =\left[\begin{array}{c}
-963000 \cos 314.2 t \\
963000 \cos 314.2 t \\
482000 \cos 314.2 t
\end{array}\right]
\end{aligned}
$$

$1093750 \bar{z}+427217148 z^{\alpha}+427217148 z-$ $963000 \cos 314.2 t$

$248554688 \ddot{\theta}+2.4030965 \times 10^{10} \theta+3.886191 \times$ $10^{10} \theta=63000 \cos 314.2 t$

$248554688 \ddot{\emptyset}+2.4030965 \times 10^{10} \dot{\emptyset}+3.886191 \times$ $10^{10} \emptyset=482000 \cos 314.2 t$ 
The solution of these equations is illustrated in Fig.8, 9 and 10

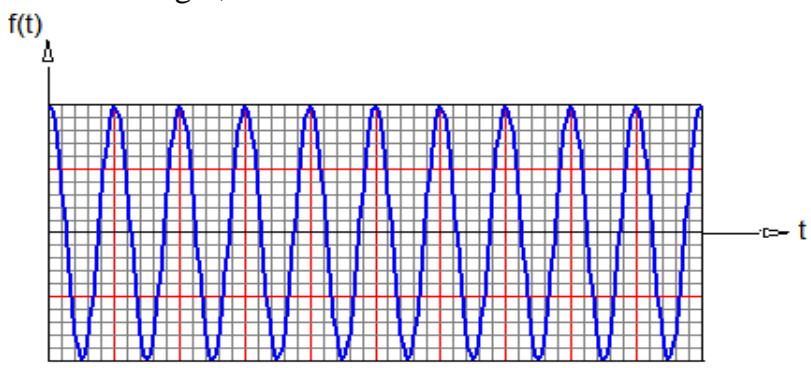

Fig. 7. Force-time relation

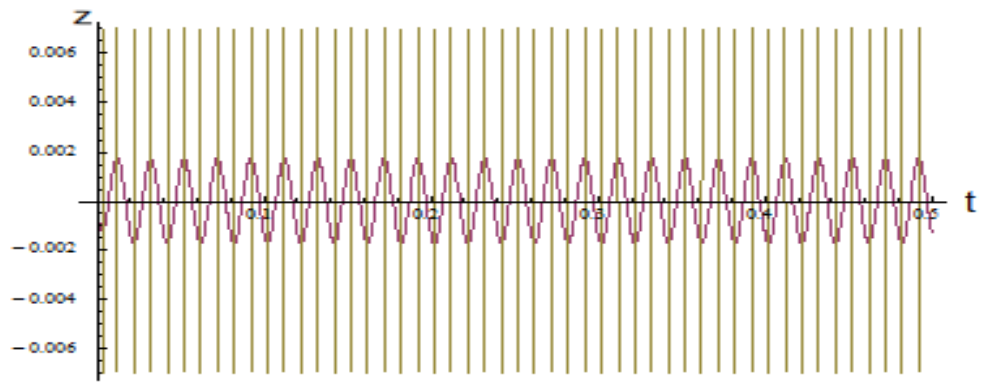

Fig. 8. Displacement in $\mathrm{z}$ direction-time relation

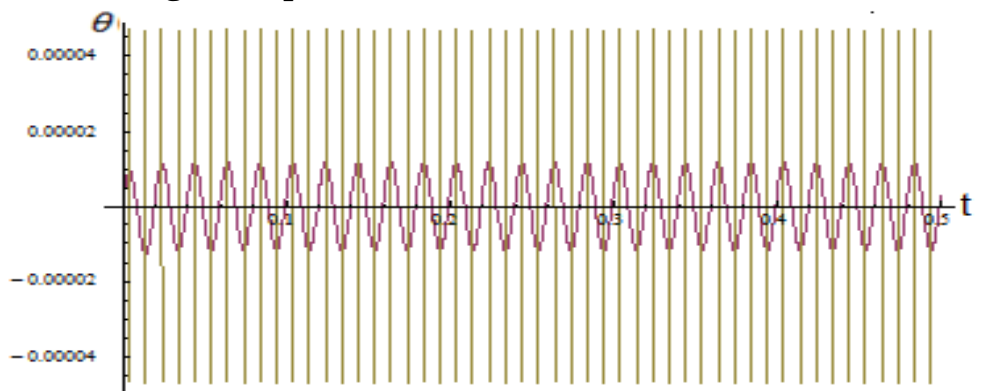

Fig. 9. Rotation about $\mathrm{x}$-axis-time relation

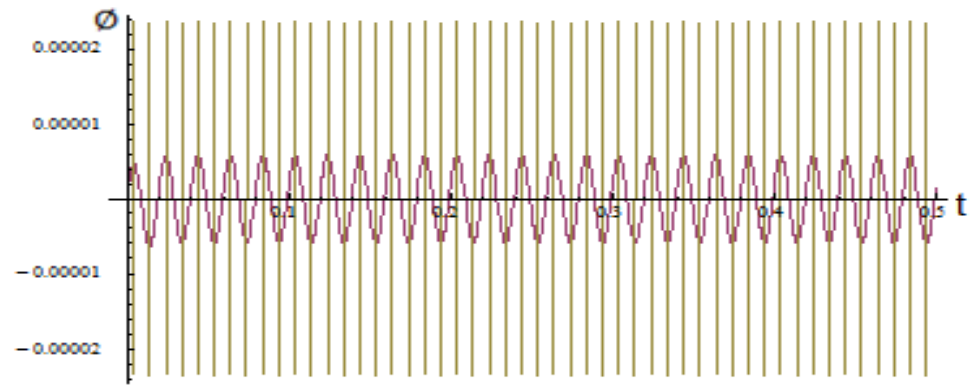

Fig. 10. Rotation about $x$-axis-time relation

\subsection{Case 2}

In this case the foundation is subjected to an eccentric periodic force and rests on a homogenous soft clay soil (the stiffness and damping at all corners are equal).

This soft clay has properties $\Phi=0, \mathrm{C}=40 \mathrm{KN} / \mathrm{m}^{2}, \rho$ $=18 \mathrm{KN} / \mathrm{m}^{2}, \mathrm{E}=15 \mathrm{Mpa}[5], \mathrm{G}=\frac{E}{2(1+\mathrm{I})}=\frac{15 \times 10^{6}}{2(1+0.5)}=5$ $M p a, \omega=1.2 \mathrm{rad} / \mathrm{sec}$

Dynamic soil properties are:

$$
\begin{aligned}
& \overline{k_{\mathrm{l}}}=n_{\mathrm{i}} \cdot k_{\mathrm{i}}, \quad \overline{c_{\mathrm{l}}}=\lambda_{\mathrm{i}} \cdot c_{\mathrm{i}}[6] \\
& k_{z}=S_{z} \frac{2 L G}{1-\pi}, S_{z}=0.73+1.54\left(l_{a}\right)^{0.75}=0.73+1.54(1)^{0.75} \\
& =2.27 \\
& k_{z}=2.27 \times \frac{2 \times 7.5 \times 50000000}{1-0.5}=340500000 \mathrm{~N} / \mathrm{m}
\end{aligned}
$$

$$
\begin{aligned}
& c_{z}=\lambda_{z} \rho \cdot V_{L A^{x}} A=1.05 \times 18000 \times 26.37 \times 15 \times 15= \\
& 112138425 \mathrm{~kg} / \mathrm{m} \\
& \overline{c_{z}}=c_{z}=112138425 \mathrm{~kg} / \mathrm{sec} \\
& k_{d}=\overline{k_{z}}-\omega \overline{c_{z}} D_{z}=306450000-1.2 \times 112138425 \times 0.005 \\
& =305777169 \mathrm{~N} / \mathrm{m} \\
& c_{d}=\overline{c_{z}}+\frac{2 \overline{k_{z}} D_{z}}{\omega}=112138425+\frac{2 \times \text { a06450000 } \times 0.005}{1.2}= \\
& 114692175 \mathrm{~kg} / \mathrm{sec} \\
& k_{d}=305777169 \mathrm{~N} / \mathrm{m} \text { and } c_{d}=114692175 \mathrm{~kg} / \mathrm{sec} \\
& \text { For machine: } \\
& \text { Weight of Machine }=105.975 \text { ton } \\
& \text { For rigid Raft foundation } \\
& \text { The dimension of the footing is } 15 \times 15, \text { assume that the } \\
& \text { net allowable stress of soil } q_{\mathrm{na}}=0.5 \mathrm{~kg} / \mathrm{cm}^{2} \text {. So take }
\end{aligned}
$$


E. A. El-Kasaby , M. D. Khedr, M. H. Mansour and Mona I. Badawi "RESPONSE OF ...."

thickness of foundation $=1.5 \mathrm{~m} \cdot[\mathrm{[}]$

1- Mass of foundation $=(337.5 \times 2500)+105975=$ $949725 \mathrm{~kg}$

2- $l_{x}$ Mass moment of inertia about $\mathrm{x}$ axis

3- $I_{y}$ Mass moment of inertia about y axis

$l_{x}=l_{y}=\frac{1}{12} m\left(y^{2}+z^{2}\right)=\frac{1}{12} 949725\left(15^{2}+1.5^{2}\right)=$ $17985418 \mathrm{~kg} \cdot \mathrm{m}^{2}$

Force properties

This force can be written in the form as a function of time as

$$
f(t)=\left\{\begin{array}{ccl}
19600000 & 0 \leq t \leq 2.5 & \sec \\
-98000000 t+294000000 & 2.5 \leq t \leq 3 & \sec [8] \\
0 & 3 \leq t \leq 5.45 & \sec
\end{array}\right.
$$

Any periodic function of time can be represented by Fourier series as an infinite sum of sine and cosine functions

If $f(t)$ is a periodic function with period $\tau=\frac{2 \pi}{\omega}$ its

Fourier series representation is given by

$F(t)=\frac{a_{0}}{2}+\sum_{j=1}^{\mathrm{m}} a_{j} \cos j \omega t+\sum_{j=1}^{\mathrm{m}} a_{j} \sin j \omega t$

Substituting in equation (51)

$$
\begin{aligned}
& F(t)=\frac{26.89 \times 10^{6}}{2}-11 \times 10^{6} \cos 1.2 t+0.25 \times \\
& 10^{6} \cos 2.4 t-1.46 \times 10^{6} \cos 3.6 t+16.6 \times \\
& 10^{6} \sin 1.2 t-8 \times 10^{6} \sin 2.4 t+4.9 \times 10^{6} \sin 3.6 t
\end{aligned}
$$

The force is eccentric from the center of the foundation $e_{x}=1 \mathrm{~m}, e_{y}=0.5 \mathrm{~m}$

Substituting in the general form equation (43)
$\left[\begin{array}{ccc}949725 & 0 & 0 \\ 0 & 17985418 & 0 \\ 0 & 0 & 17985418\end{array}\right]\left[\begin{array}{l}\tilde{z} \\ \ddot{\theta} \\ \ddot{\emptyset}\end{array}\right]$

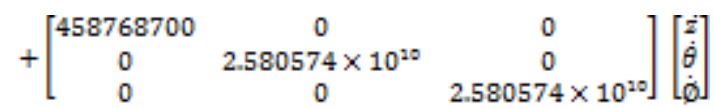

$+\left[\begin{array}{ccc}1223108676 & 0 & 0 \\ 0 & 6.879986 \times 10^{20} & 0 \\ 0 & 0 & 6.879986 \times 10^{10}\end{array}\right] \|\left[\begin{array}{l}\tilde{z} \\ \theta \\ 0\end{array}\right]$

$=\left[\begin{array}{l}-p_{t} \\ M_{y} \\ M_{y}\end{array}\right]$

$949725 \mathrm{z}+458768700 z^{\circ}+1223108676 z=$ $-\left(\frac{26.89 \times 10^{6}}{2}-11 \times 10^{6} \cos 1.2 t+0.25 \times 10^{6} \cos 2.4 t-\right.$ $1.46 \times 10^{6} \cos 3.6 t+16.6 \times 10^{6} \sin 1.2 t-8 \times$ $10^{6} \sin 2.4 t+4.9 \times 10^{6} \sin 3.6 t$

$17985418 \theta * 2.580574 \times 10^{10} \theta^{*}$

$+6.879986 \times 10^{10} \theta=\left(\frac{26.89 \times 10^{6}}{2}-\right.$

$11 \times 10^{6} \cos 1.2 t+0.25 \times 10^{6} \cos 2.4 t-1.46 \times$

$10^{6} \cos 3.6 t+16.6 \times 10^{6} \sin 1.2 t-8 \times 10^{6} \sin 2.4 t+$ $4.9 \times 10^{6} \sin 3.6 t$

)

$17985418 \emptyset$

$+2.580574 \times 10^{10} \emptyset+6.879986 \times 10^{10} \emptyset=\frac{1}{2}$

$\frac{26.89 \times 10^{6}}{2}-11 \times 10^{6} \cos 1.2 t+0.25 \times 10^{6} \cos 2.4 t-$

$1.46 \times 10^{6} \cos 3.6 t+16.6 \times 10^{6} \sin 1.2 t-8 \times$

$10^{6} \sin 2.4 t+4.9 \times 10^{6} \sin 3.6 t$

)

The solution of these equations is illustrated in Fig. 12, 13 and 14.

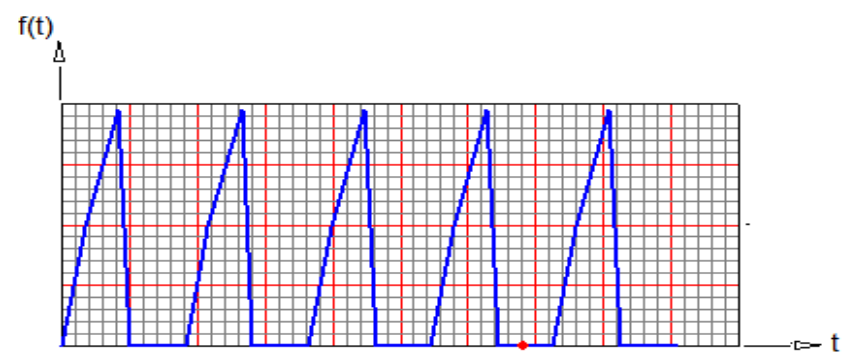

Fig. 11.Force-time relationship

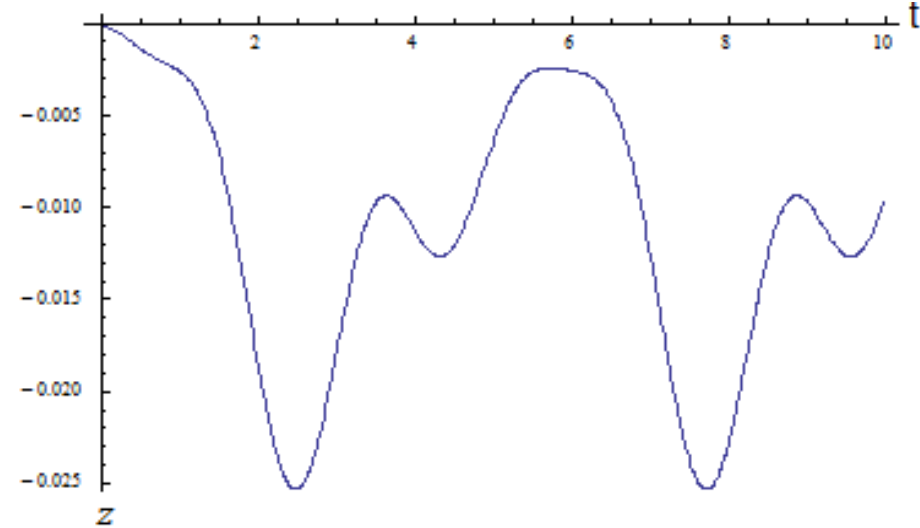

Fig. 12. Displacement in z direction-time relation 


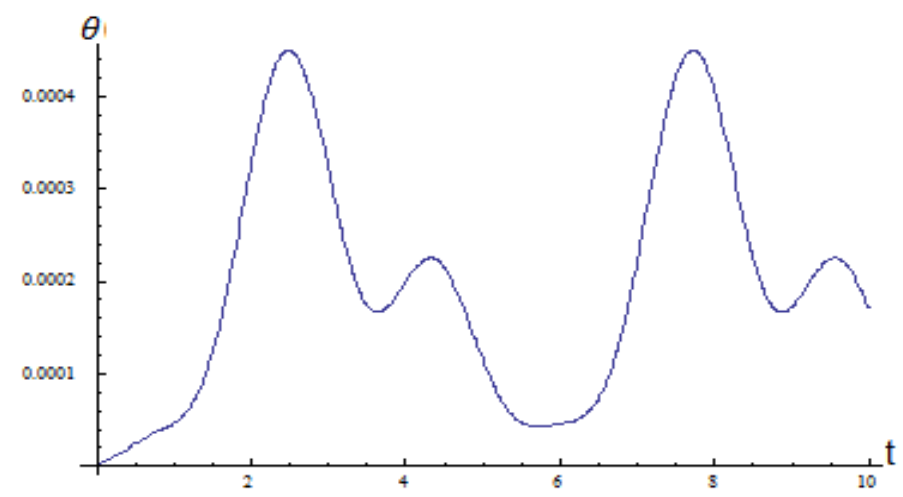

Fig. 13. Rotation about $x$-axis-Time relation

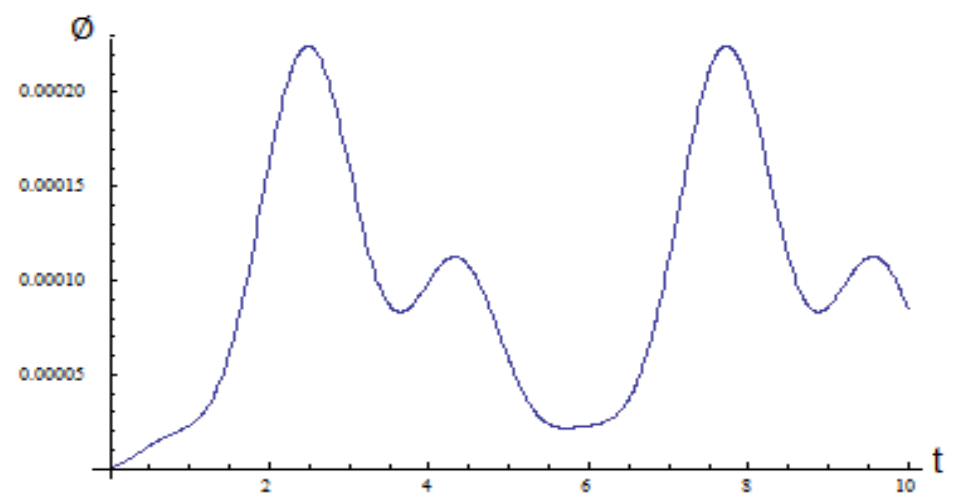

Fig. 14. Rotation about $x$-axis-time relation

\subsection{Case 3}

This case the foundation is subjected to an eccentric impulsive force and rests on a homogenous stiff clay soil (the stiffness and damping at all corners are equal). This stiff clay has properties $\Phi=0, \quad \mathrm{C}=40 \mathrm{KN} / \mathrm{m}^{2}, \quad \rho$ $=18 \mathrm{KN} / \mathrm{m}^{2}, \mathrm{E}=45 \mathrm{Mpa}$ [5]

$\mathrm{G}=\frac{E}{2(1+\mathrm{m})}=\frac{45 \times 10^{6}}{2(1+0.5)}=15 \mathrm{Mpa}, \omega=63.5 \mathrm{rad} / \mathrm{sec}$

Dynamic soil properties are:

$$
\begin{aligned}
& \overline{k_{\mathrm{u}}}=n_{\mathrm{i}} \cdot k_{\mathrm{i}} \quad, \quad \overline{c_{\mathrm{l}}}=\lambda_{\mathrm{i}} \cdot c_{\mathrm{i}}[6] \\
& k_{z}=S_{z} \frac{2 L G}{1-\Pi}, \quad S_{z}=0.73+1.54\left(J_{a}\right)^{0.75}= \\
& 0.73+1.54(1)^{0.75}=2.27 \\
& k_{z}=2.27 \times \frac{2 \times 7.5 \times 15000000}{1-0.5}=1021500000 \mathrm{~N} / \mathrm{m} \\
& \overline{k_{z}}=1021500000 \times 0.7=715050000 \mathrm{~N} / \mathrm{m} \\
& c_{z}=\rho \cdot V_{L A} \cdot A \quad=18000 \times 62.485 \times 15 \times 15= \\
& 253064250 \mathrm{~kg} / \mathrm{m} \quad \\
& \overline{c_{z}}=c_{z}=253064250 \mathrm{~kg} / \mathrm{sec} \\
& k_{d}=\overline{k_{z}}-\omega \overline{c_{z}} D_{z}=715050000-63.5 \times 253064250 \times \\
& 0.005=634702100 \mathrm{~N} / \mathrm{m} \\
& c_{d}=\overline{c_{z}}+\frac{2 \overline{k_{z}} D_{z}}{\omega}=253064250+\frac{2 \times 715050000 \times 0.005}{66.5}= \\
& 253176856 \mathrm{~kg} / \mathrm{sec} \\
& k_{d d}=634702100 \mathrm{~N} / \mathrm{m}, c_{d}=253176856 \mathrm{~kg} / \mathrm{sec}
\end{aligned}
$$

For machine:

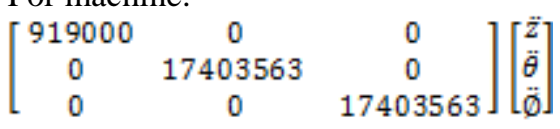

Weight of Machine $=75$ ton

For rigid Raft foundation :

The dimension of the footing is $15 \times 15$, assume that the net allowable stress of soil $q_{n a}=1.2 \mathrm{~kg} / \mathrm{cm}^{2}$. So take thickness of foundation $=1.5 \mathrm{~m}$

1- Mass of foundation $=(337.5 \times 2500)+75=919$ ton

2- $I_{x}$ Mass moment of inertia about $\mathrm{x}$ axis

$$
l_{x}=\frac{1}{12} m\left(y^{2}+z^{2}\right)=\frac{1}{12} 919000\left(15^{2}+1.5^{2}\right)=
$$

$17403563 \mathrm{~kg} \cdot \mathrm{m}^{2}$

3- $I_{y}$ Mass moment of inertia about y axis

$$
l_{y}=\frac{1}{12} m\left(y^{2}+z^{2}\right)=\frac{1}{12} 919000\left(15^{2}+1.5^{2}\right)=
$$

$17403563 \mathrm{~kg} \cdot \mathrm{m}^{2}$

Force properties

It is a half-sine pulse load. This force can be written in the form as a function of time as

$f(t)=\left\{\begin{array}{cc}P_{g} \sin \left(\frac{\pi}{t_{p}} t\right)=P_{g} \sin (\omega t), & 0<t<t_{P}[9] \\ 0_{s} & t>t_{p}\end{array}\right.$

Where

$P_{g}=\frac{P_{r} \pi}{2}, P_{r}=1 \mathrm{~N}, \omega=\frac{\pi}{t_{F}}=63.5 \mathrm{rad} / \mathrm{sec}$.

The force is eccentric from the center of the foundation $e_{x}=1 \mathrm{~m}, e_{y}=.5 \mathrm{~m}$

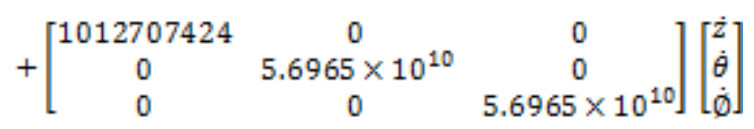


E. A. El-Kasaby, M. D. Khedr, M. H. Mansour and Mona I. Badawi " RESPONSE OF ..."

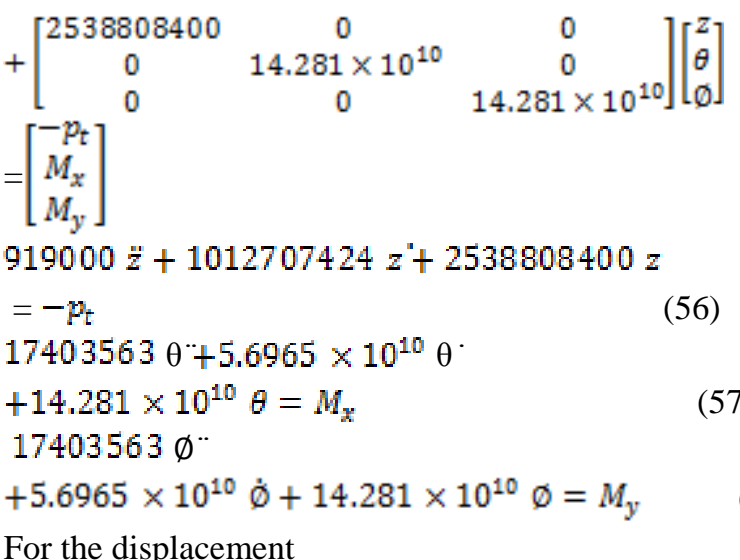

For the displacement

$\omega_{n}=\sqrt{\frac{2538908400}{919000}}=52.56, \omega_{n}^{d}=52.56 \sqrt{1-0.1048^{2}}=$

52.27

$z(t)=$

$-43.162 \cos 63.5 t+61.97 \sin 63.5 t+$

$43.162 e^{-5.51 t} \cos 52.27 t$

$+3.1 \times 56.63^{52.27 t} e^{-5.51 t} \sin 52.27 t$

(59)

For rotation about $\mathrm{x}$-axis

$$
\begin{aligned}
& \omega_{n}=\sqrt{\frac{14.281 \times 10^{10}}{1740356 a}}=90.58, \\
& \omega_{n}^{s}=90.586 \sqrt{1-0.1807^{2}}=89.1 \\
& \theta(t)= \\
& -86.44 \cos 63.5 t-2.75 \sin 63.5 t+ \\
& 86.44 e^{-16.27 t} \cos 90.586 t
\end{aligned}
$$$$
+2.64 \times 40.26^{89.1 t} e^{-16.27 t} \sin 89.1 t
$$

For rotation about y-axis

$$
\begin{aligned}
& \omega_{n}=\sqrt{\frac{14.281 \times 10^{10}}{1740156 a}}=90.586, \\
& \omega_{n}^{s}=90.586 \sqrt{1-0.1807^{2}}=89.1 \\
& \emptyset(t)= \\
& -43.22 \cos 63.5 t-1.375 \sin 63.5 t+ \\
& 43.22 e^{-16.27 t} \cos 90.586 t
\end{aligned}
$$$$
+1.32 \times 40.26^{89.1 t} e^{-16.27 t} \sin 89.1 t
$$

The solution of these equations is illustrated in Fig. 15, 16 and 17.

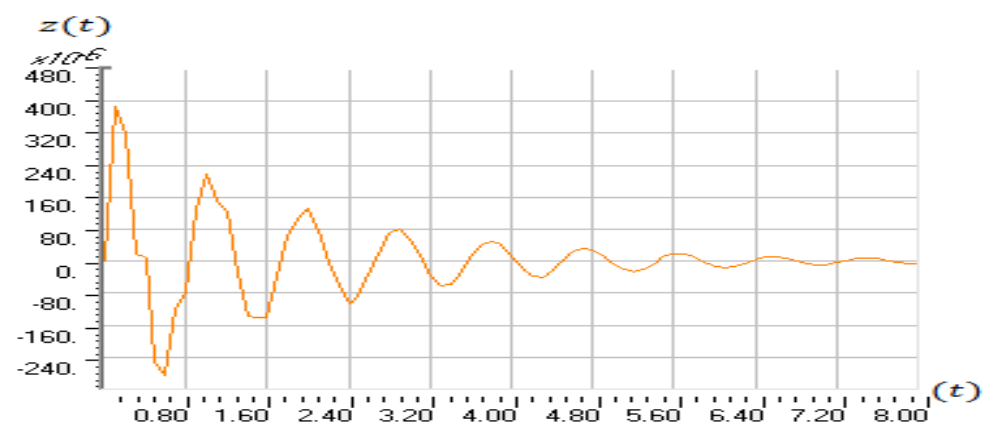

Fig. 15. Displacement in $\mathrm{z}$ direction-time relation

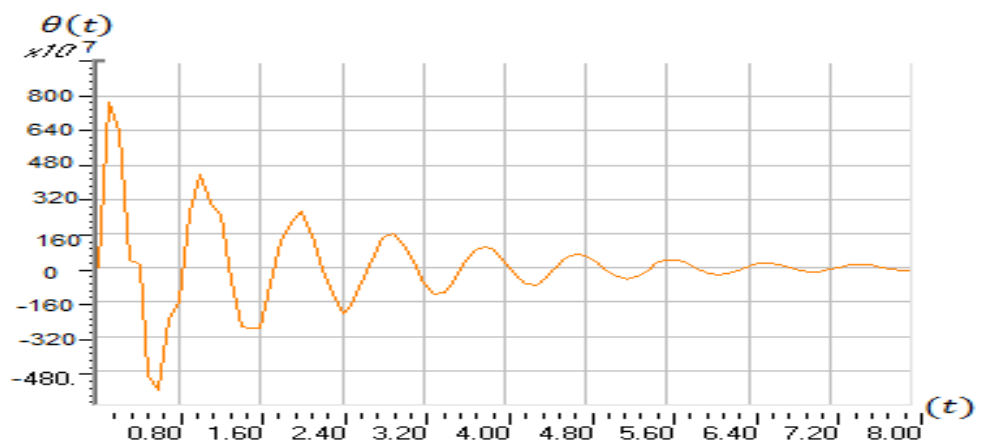

Fig. 16. Rotation about $x$-axis-time relation

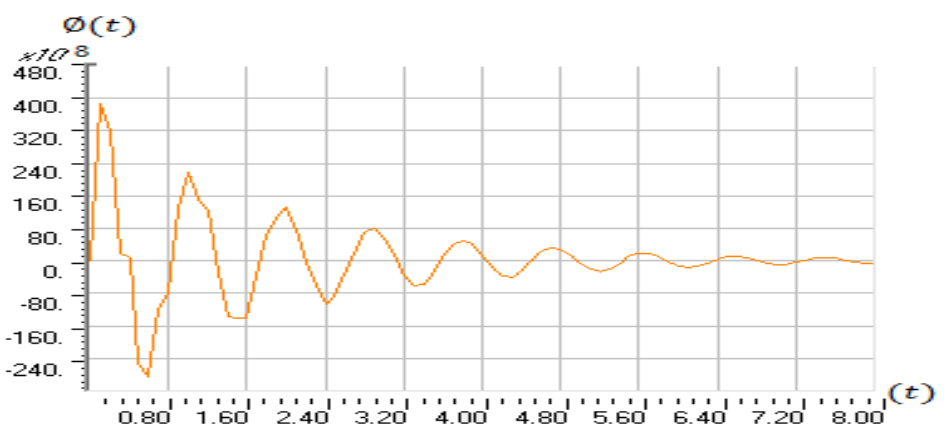

Fig. 17. Rotation about $x$-axis-time relation 


\section{Conclusion:}

Machine-raft foundation-soil system is modeled as a three degree of freedom system, translation in the vertical direction, ( $\mathrm{z}$ direction), and rotation about $\mathrm{x}$ axis and $\mathrm{y}$ axis. The dynamic stiffness and damping properties of the soil are determined according to the existing force and the type of soil. The relation between the dynamic stiffness and damping with the shear modulus is determined. The apparent mass of the rigid raft is calculated according to the assumed dimensions. The response of the rigid foundation to an eccentric (harmonic, periodic, impulse) load is calculated by using Mathematica program. The relation between the response (displacement, rotation about $\mathrm{x}$-axis and rotation about $\mathrm{y}$ axis) with time is drawn. The harmonic load is represented by the relation between forces with time (Sine-function). The periodic force is represented by Fourier expansion using the first 6 harmonics of the expansion. The impact force is represented by the integration of the force as a function of time. Three cases are studied. The effect of changing the type of soil and the type of load on the response is studied. Also the following conclusions are presented:

- The response of the raft foundation rested on homogeneous soft clay subjected to the load is greater than in stiff clay case.

- The relation between the response and dynamic stiffness is inversely proportional.

- The Relation between the dynamic stiffness and the shear modulus is directly proportional. Thus, the relation between the response and the shear modulus is inversely proportional. Also the response of system decreases with increasing of soil strength.

\section{References:}

1. Rao Singiresu, S. "Mechanical Vibrations" Fifth Edition, Pearson Education, Inc, publishing as Prentice Hall, 1 Lake Street, Upper Saddle River, NJ 07458, (2011).

2. Bhatia, K.G. "Machine Foundation in Power Plant and Other Industries-Case Studies", Proceedings of the International Conference on case Histories in Geotechnical Engineering, St.Louis, U.S.A., Vol.2, pp.775-779, (1984).

3. Das Braja, M., Ramana, G.V. "Principle of Soil Dynamics", Gengage Learning, (2011).

4. Chopra, A.K. " Dynamic of Structures " PrenticeHall International Series In Civil Engineering Mechanics, New Jersey, USA, (1995).

5. El-Kasaby, E.A." Soil Mechanics " Dar El-Kotob El-Elmia, Fifth Edition, Cairo, Egypt (In Arabic Language), (2015).

6. Bowles, J.E. "Foundation Analysis And Design" Fifth Edition, the McGraw-Hill Companies, Inc. (1997)

7. "The Egyptian Code For Soil Mechanics and Design of Foundation" , Ministry of Housing, Utilities and Urban Communities, Code number 202, (2001).

8. Mosa, B.E. "Dynamic of Bases and Foundations", Thesis, Benha Faculty of Engineering, Egypt, (2015).

9. Chehab, A.G., El-Naggar, M.H. "Response of Block Foundations to Impact Loads", Journal of Sound and Vibration 276 293-310, (2004). 
E. A. El-Kasaby , M. D. Khedr, M. H. Mansour and Mona I. Badawi "RESPONSE OF ...." 